\title{
A well-posedness theory in measures for some kinetic models of collective motion
}

\author{
José A. CañIzo* José A. Carrillo ${ }^{\dagger}$ Jesús Rosado
}

April 12th 2010

\begin{abstract}
We present existence, uniqueness and continuous dependence results for some kinetic equations motivated by models for the collective behavior of large groups of individuals. Models of this kind have been recently proposed to study the behavior of large groups of animals, such as flocks of birds, swarms, or schools of fish. Our aim is to give a well-posedness theory for general models which possibly include a variety of effects: an interaction through a potential, such as a short-range repulsion and long-range attraction; a velocity-averaging effect where individuals try to adapt their own velocity to that of other individuals in their surroundings; and selfpropulsion effects, which take into account effects on one individual that are independent of the others. We develop our theory in a space of measures, using mass transportation distances. As consequences of our theory we show also the convergence of particle systems to their corresponding kinetic equations, and the local-in-time convergence to the hydrodynamic limit for one of the models.
\end{abstract}

Keywords:kinetic theory, measure solutions, interacting particle systems, MongeKantorovich-Rubinstein distance, stability, particle methods, swarming.

AMS Subject Classification: 35A05, 35B40, 82D99, 92D50.

\footnotetext{
*Departament de Matemàtiques, Universitat Autònoma de Barcelona, 08193 Bellaterra, Spain. E-mail: canizo@mat.uab.es

${ }^{\dagger}$ ICREA - Departament de Matemàtiques, Universitat Autònoma de Barcelona, 08193 Bellaterra, Spain. E-mail: carrillo@mat.uab.es

¥Departament de Matemàtiques, Universitat Autònoma de Barcelona, 08193 Bellaterra, Spain. E-mail: jrosado@mat.uab.es
} 


\section{Introduction}

The description of the collective motion (swarming) of multi-agent aggregates resulting into large-scale structures is a striking phenomena, as illustrated by the examples provided by birds, fish, bees or ants. Explaining the emergence of these coordinated movements in terms of microscopic decisions of each individual member of a swarm is a hot matter of research in the natural sciences [6, 11, 29. The formation of swarms and milling or flocking patterns have been reported in animals with highly developed social organization like insects (locusts, bees, ants, ...) [11, fishes [1, 2] and birds [6, 29] but also in micro-organisms as myxobacteria [22]. Moreover, the understanding of natural swarms has been used as an engineering design principle for unmanned artificial robots operation [5, 30.

The physics and applied mathematics literature has proliferated and sprung in this direction in the recent years trying to model these phenomena, mainly based on two strategies of description: individual-based models or particle dynamics [35, 29, 23, 6, 26, 18, 11, 15, 12, 13, 24, 25] and continuum models based on PDEs for the density or for the momentum of the particle ensemble [29, 33, 34, 10, 16]. The key feature to explain is the emergence of selforganization: flocking, milling, double milling patterns or other coherent behavior.

Particle descriptions usually include three basic mechanisms in different regions: short-range repulsion zone, long-range attraction zone and alignment or orientation zone, leading to the so-called three-zone models. In addition, some of them incorporate a mechanism for establishing a fixed asymptotic speed/velocity vector of agents, as is usually observed in nature. Some of the models only consider the orientation vector and not the speed in their discrete version. The main differences of all these models reside in how these three interactions are specifically considered. We will mainly work with two generic examples in which several of the effects above are included, namely the model for self-propelled interacting particles introduced by D'Orsogna et al in [15] and the model of alignment proposed by Cucker and Smale [12, 13].

Together with particle and continuum models based on macroscopic densities, there has been a very recent trend of mesoscopic models by means of kinetic equations for swarming [20, 7, 19, 8]. In these models one works with a statistical description of the interacting agent system. Let us represent by $x \in \mathbb{R}^{d}$ the position, where $d \geq 1$ stands for the physical space dimension, and by $v \in \mathbb{R}^{d}$ the velocity. We are interested in studying the evolution of $f=f(t, x, v)$ representing the probability measure/density of individuals at position $x$, with velocity $v$, and at time $t \geq 0$. These are the models we study in the present paper. Given that we cover a variety of them, we refer the reader to Section 2 for a more detailed presentation of the equations.

These kinetic models bridge the particle description of swarming to the hydrodynamic one as already discussed in [20, 7, 19. The main key idea is that solutions to particle systems are in fact atomic-measure solutions for the kinetic equations, and solutions to the hydrodynamic equations are solutions of a special form to the kinetic equation; see Section 5.2 for more details. 
In some cases, suitable compactness arguments based on the stability properties in distances between probability measures allow to construct a wellposedness theory for a kinetic equation. Such an approach was done for the Vlasov equation in classical kinetic theory [27, 4, 14, 31 with several nice reviews in 28, 32, 17. Of these references, [14 uses the Monge-Kantorovich-Rubinstein distance (the one we use in the present paper); the others, as well as the recent work [19] for the kinetic Cucker-Smale model, use an approach based on the bounded Lipschitz distance.

In this paper we present a generic approach to the well-posedness of many of these models in the set of probability measures in phase space based on the modern theory of optimal transport [36]. In fact, we will use the well-known Monge-Kantorovich-Rubinstein distance between probability measures instead of the bounded Lipschitz distance. Its better duality properties actually make this technical approach easier in terms of estimates leading to one of our crucial results: a stability property of solutions to swarming equations under quite general conditions.

We derive some consequences from this stability estimate. First, we prove the mean-field limit, or convergence of the particle method towards a measure solution of the kinetic equation. This mean field limit is then established without any resorting to the BBGKY hierarchy or the molecular chaos hypothesis [3, 7. 19. Second, we show the stability for arbitrary times of the hydrodynamic solutions, assuming they exist, although with constants depending on time. Finally, the stability result can be used to obtain qualitative properties of the measure solutions of the kinetic equations, as it has been done in 8 for the kinetic Cucker-Smale model.

This strategy is quite general, and we first demonstrate its use in a particular kinetic model introduced in 7 for dealing with the mesoscopic description and certain patterns not covered by the particle model proposed in [15. Other models are treated by the same procedure in subsequent sections, as the kinetic Cucker-Smale model proposed in 20] for the original alignment mechanism in [12, 13, the models studied in [24, 25], or any linear combination of these mechanisms. We finally give general conditions on a model that are sufficient for our well-posedness results to be valid.

Let us comment on some limitations of the method we use. The first one is that, as we work with solutions in a weak measure sense, we have to require our interaction terms to be locally Lipschitz in order in order to carry out the theory. This is a well-known limitation in the literature for working with the mean field limit and measure solutions, see 32, 21 and the references therein. A less fundamental one is that we always work with compactly supported solutions. One could probably develop a theory substituting this condition by a suitable control on moments of the solution, and then adapting the estimates to this setting; however, in the present paper we do not pursue further extensions in this direction.

Next section does a simple and brief review of the main interacting particle systems under analysis and the needed concepts for the Monge-KantorovichRubinstein distance between probability measures. The third section is devoted 
to the proof of the main result of existence, uniqueness and stability of measure solutions to the particular swarming equations introduced in [7. Section 4 generalizes this approach to a general family of these equations. Finally, section 5 draws some consequences of the stability property: the convergence of the particle method and the mean-field limit are proved for the general model, while the stability in a finite time interval of hydrodynamic solutions is shown for the swarming model used in Section 3 .

\section{Preliminaries}

In this section we introduce the models mentioned in the introduction. We give some particular representative cases and specify the models to which our results apply. Also, we recall some notions about optimal transport that shall come in handy.

\subsection{Main Kinetic Models}

The particle model proposed in [15] reads as:

$$
\begin{cases}\frac{d x_{i}}{d t}=v_{i}, & (i=1, \ldots, N) \\ \frac{d v_{i}}{d t}=\left(\alpha-\beta\left|v_{i}\right|^{2}\right) v_{i}-\frac{1}{N} \sum_{j \neq i} \nabla U\left(\left|x_{i}-x_{j}\right|\right), & (i=1, \ldots, N) .\end{cases}
$$

where $\alpha, \beta$ are nonnegative parameters, $U: \mathbb{R}^{d} \longrightarrow \mathbb{R}$ is a given potential modeling the short-range repulsion and long-range attraction typical in these models, and $N$ is the number of particles. Here, the potential has been scaled depending on the mass of each particle as in [7], where we refer for further discussion. The term corresponding to $\alpha$ models the self-propulsion of individuals, whereas the term corresponding to $\beta$ is the friction assumed to follow Rayleigh's law. The balance of these two terms imposes an asymptotic speed to the agent (if other effects are ignored), but does not influence the orientation vector. A typical choice for $U$ is the Morse potential which is radial and given by

$$
U(x)=k(|x|) \quad \text { with } \quad k(r)=-C_{A} e^{-r / \ell_{A}}+C_{R} e^{-r / \ell_{R}},
$$

where $C_{A}, C_{R}$ and $\ell_{A}, \ell_{R}$ are the strengths and the typical lengths of attraction and repulsion, respectively. This potential does not satisfy the smoothness assumption in our main theorems but the qualitative behavior of the particle system does not depend on this particular fact [15]. In fact, a typical potential satisfying all of our hypotheses is

$$
U(x)=-C_{A} e^{-|x|^{2} / \ell_{A}^{2}}+C_{R} e^{-|x|^{2} / \ell_{R}^{2}} .
$$

The kinetic equation associated to this particle model as discussed in [7] gives the evolution of $f=f(t, x, v)$ as

$$
\partial_{t} f+v \cdot \nabla_{x} f-(\nabla U * \rho) \cdot \nabla_{v} f+\operatorname{div}_{v}\left(\left(\alpha-\beta|v|^{2}\right) v f\right)=0
$$


where $\rho$ represents the macroscopic density of $f$ :

$$
\rho(t, x):=\int_{\mathbb{R}^{d}} f(t, x, v) d v \quad \text { for } t \geq 0, x \in \mathbb{R}^{d} .
$$

In the Cucker-Smale model, introduced in [12, 13, the only mechanism taken into account is the reorientation interaction between agents. Each agent in the swarm tries to mimick other individuals by adjusting/averaging their relative velocity with all the others. This averaging is weighted in such a way that closer individuals have more influence than further ones. For a system with $N$ individuals the Cucker-Smale model reads as

$$
\left\{\begin{array}{l}
\frac{d x_{i}}{d t}=v_{i} \\
\frac{d v_{i}}{d t}=\frac{1}{N} \sum_{j=1}^{N} w_{i j}\left(v_{j}-v_{i}\right),
\end{array}\right.
$$

with the communication rate $w(x)$ given by:

$$
w_{i j}=w\left(\left|x_{i}-x_{j}\right|\right)=\frac{1}{\left(1+\left|x_{i}-x_{j}\right|^{2}\right)^{\gamma}}
$$

for some $\gamma \geq 0$. This particle model leads to the following kinetic model [20, [19, 8]:

$$
\frac{\partial f}{\partial t}+v \cdot \nabla_{x} f=\nabla_{v} \cdot[\xi[f] f]
$$

where $\xi[f](x, v, t)=(H * f)(x, v, t)$, with $H(x, v)=w(x) v$ and $*$ standing for the convolution in both position and velocity $(x$ and $v)$. We refer to [12, 13, 8] for further discussion about this model and qualitative properties.

Moreover, quite general models incorporating the three effects previously discussed have been considered in [24, 25]. In particular, they consider that $N$ individuals follow the system:

$$
\left\{\begin{array}{l}
\frac{d x_{i}}{d t}=v_{i} \\
\frac{d v_{i}}{d t}=F_{i}^{A}+F_{i}^{I}
\end{array}\right.
$$

where $F_{i}^{A}$ is the self-propulsion autonomously generated by the $i$ th-individual, while $F_{i}^{I}$ is due to interaction with the others. The model in Section 3 corresponds to $F_{i}^{A}=\left(\alpha-\beta\left|v_{i}\right|^{2}\right) v_{i}$, while the term $F_{i}^{A}=-\beta v_{i}$ is considered in [23], and $F_{i}^{A}=a_{i}-\beta v_{i}$ in [24, 25]. Here, $a_{i}$ is an autonomous self-propulsion force generated by the $i$ th-particle, and may depend on environmental influences and the location of the particle in the school. The interaction with other individuals can be generally modeled as:

$$
F_{i}^{I}=F_{i}^{I, x}+F_{i}^{I, v}=\sum_{j=1}^{N} g_{ \pm}\left(\left|x_{i}-x_{j}\right|\right) \frac{x_{j}-x_{i}}{\left|x_{i}-x_{j}\right|}+\sum_{j=1}^{N} h_{ \pm}\left(\left|v_{i}-v_{j}\right|\right) \frac{v_{j}-v_{i}}{\left|v_{i}-v_{j}\right|} .
$$


Here, $g_{+}$and $h_{+}\left(g_{-}\right.$and $\left.h_{-}\right)$are chosen when the influence comes from the front (behind), i.e., if $\left(x_{j}-x_{i}\right) \cdot v_{i}>0(<0)$; choosing $g_{+} \neq g_{-}$and $h_{+} \neq h_{-}$ means that the forces from particles in front and those from particles behind are different. The sign of the functions $g_{ \pm}(r)$ encodes the short-range repulsion and long-range attraction for particles in front of $(+)$ and behind (-) the $i$ thparticle. Similarly, $h_{+}>0(<0)$ implies that the velocity-dependent force makes the velocity of particle $i$ get closer to (away from) that of particle $j$.

In the next sections we will be concerned with the well-posedness for measure solutions to (1), (3) and generalized kinetic equations including the corresponding to the $N$-individuals model in (4).

\subsection{Preliminaries on mass transportation and notation}

Let us recall some notation and known results about mass transportation that we will use in the next sections. For a more detailed approach, the interested reader can refer to 9,36 .

We consider the space of probability measures $\mathcal{P}_{1}\left(\mathbb{R}^{d}\right)$, consisting of all probability measures on $\mathbb{R}^{d}$ with finite first moment. In $\mathcal{P}_{1}\left(\mathbb{R}^{d}\right)$ a natural concept of distance to work with is the so-called Monge-Kantorovich-Rubinstein distance,

$$
W_{1}(f, g)=\sup \left\{\left|\int_{\mathbb{R}^{d}} \varphi(P)(f(P)-g(P)) d P\right|, \varphi \in \operatorname{Lip}\left(\mathbb{R}^{d}\right), \operatorname{Lip}(\varphi) \leq 1\right\},
$$

where $\operatorname{Lip}\left(\mathbb{R}^{d}\right)$ denotes the set of Lipschitz functions on $\mathbb{R}^{d}$ and $\operatorname{Lip}(\varphi)$ the Lipschitz constant of a function $\varphi$. Denoting by $\Lambda$ the set of transference plans between the measures $f$ and $g$, i.e., probability measures in the product space $\mathbb{R}^{d} \times \mathbb{R}^{d}$ with first and second marginals $f$ and $g$ respectively, then we have

$$
W_{1}(f, g)=\inf _{\pi \in \Lambda}\left\{\int_{\mathbb{R}^{d} \times \mathbb{R}^{d}}\left|P_{1}-P_{2}\right| d \pi\left(P_{1}, P_{2}\right)\right\}
$$

by Kantorovich duality. $\mathcal{P}_{1}\left(\mathbb{R}^{d}\right)$ endowed with this distance is a complete metric space. In the following proposition we recall some of its properties. We refer to [36] for a survey of these basic facts.

Proposition 2.1 ( $W_{1}$-properties). The following properties of the distance $W_{1}$ hold:

i) Optimal transference plan: The infimum in the definition of the distance $W_{1}$ is achieved. Any joint probability measure $\Pi_{o}$ satisfying:

$$
W_{1}(f, g)=\int_{\mathbb{R}^{d} \times \mathbb{R}^{d}}\left|P_{1}-P_{2}\right| d \Pi_{o}\left(P_{1}, P_{2}\right) .
$$

is called an optimal transference plan and it is generically non unique for the $W_{1}$-distance.

ii) Convergence of measures: Given $\left\{f_{k}\right\}_{k \geq 1}$ and $f$ in $\mathcal{P}_{1}\left(\mathbb{R}^{d}\right)$, the following three assertions are equivalent: 
a) $W_{1}\left(f_{k}, f\right)$ tends to 0 as $n$ goes to infinity.

b) $f_{k}$ tends to $f$ weakly-* as measures as $k$ goes to infinity and

$$
\sup _{k \geq 1} \int_{|v|>R}|v| f_{k}(v) d v \rightarrow 0 \text { as } R \rightarrow+\infty .
$$

c) $f_{k}$ tends to $f$ weakly-* as measures and

$$
\int_{\mathbb{R}^{d}}|v| f_{k}(v) d v \rightarrow \int_{\mathbb{R}^{d}}|v| f(v) d v \text { as } n \rightarrow+\infty .
$$

Throughout the paper we will denote the integral of a function $\varphi=\varphi(x)$ with respect to a measure $\mu$ by $\int \varphi(x) \mu(x) d x$, even if the measure is not absolutely continuous with respect to Lebesgue measure, and hence does not have an associated density.

Given a probability measure $f \in \mathcal{P}_{1}\left(\mathbb{R}^{d} \times \mathbb{R}^{d}\right)$ we always denote by $\rho$ its first marginal, written as follows by an abuse of notation:

$$
\rho(x):=\int_{\mathbb{R}^{d}} f(x, v) d v .
$$

To be more precise, $\rho$ is given by its action on a $\mathcal{C}_{c}^{0}$ function $\phi: \mathbb{R}^{d} \rightarrow \mathbb{R}$,

$$
\int_{\mathbb{R}^{d}} \rho(x) \phi(x) d x=\int_{\mathbb{R}^{d} \times \mathbb{R}^{d}} f(x, v) \phi(x) d x d v .
$$

For $T>0$ and a function $f:[0, T] \rightarrow \mathcal{P}_{1}\left(\mathbb{R}^{d} \times \mathbb{R}^{d}\right)$, it is understood that $\rho$ is the function $\rho:[0, T] \rightarrow \mathcal{P}_{1}\left(\mathbb{R}^{d}\right)$ obtained by taking the first marginal at each time $t$. Whenever we need to indicate explicitly the dependence of $\rho$ on $f$, we write $\rho[f]$ instead of just $\rho$.

We denote by $B_{R}$ the closed ball with center 0 and radius $R>0$ in the Euclidean space $\mathbb{R}^{n}$ of some dimension $n$. When we need to explicitly indicate the dimension of the space, we will write $B_{R}^{n}$. For a function $H: \mathbb{R}^{n} \rightarrow \mathbb{R}^{m}$, we will write $\operatorname{Lip}_{R}(H)$ to denote the Lipschitz constant of $H$ in the ball $B_{R} \subseteq \mathbb{R}^{n}$. For $T>0$ and a function $H:[0, T] \times \mathbb{R}^{n} \rightarrow \mathbb{R}^{m}, H=H(t, x)$, we again write $\operatorname{Lip}_{R}(H)$ to denote the Lipschitz constant with respect to $x$ of $H$ in the ball $B_{R} \subseteq \mathbb{R}^{n}$; this is, $\operatorname{Lip}_{R}(H)$ is the smallest constant such that

$$
\left|H\left(t, x_{1}\right)-H\left(t, x_{2}\right)\right| \leq \operatorname{Lip}_{R}(H)\left|x_{1}-x_{2}\right| \quad \text { for all } x_{1}, x_{2} \in B_{R}, t \in[0, T] .
$$

For any such function $H$, we will denote the function depending on $x$ at a fixed time $t$ by $H_{t}$; this is, $H_{t}(x):=H(t, x)$.

\section{Well-posedness for a system with interaction and self-propulsion}

In this section we consider eq. (11). In this model (and in fact, in every model considered in this paper) the total mass is preserved, and by rescaling the equation and adapting the parameters suitably one easily sees that we can normalize 
the equation and consider only solutions with total mass 1 . We will do so and reduce ourselves to work with probability measures.

\subsection{Notion of solution}

In order to motivate our definition of solution to equation (1) let us consider for a moment a general field $E$ instead of $-\nabla U * \rho$. Precisely, fix $T>0$ and a function $E:[0, T] \times \mathbb{R}^{d} \rightarrow \mathbb{R}^{d}$ such that:

Hypothesis 3.1 (Conditions on $E$ ). $\quad$ 1. E is continuous on $[0, T] \times \mathbb{R}^{d}$,

2. For some $C_{E}>0$,

$$
|E(t, x)| \leq C_{E}(1+|x|), \quad \text { for all } t, x \in[0, T] \times \mathbb{R}^{d}, \text { and }
$$

3. $E$ is locally Lipschitz with respect to $x$, i.e., for any compact set $K \subseteq \mathbb{R}^{d}$ there is some $L_{K}>0$ such that

$$
|E(t, x)-E(t, y)| \leq L_{K}|x-y|, \quad t \in[0, T], \quad x, y \in K .
$$

We consider the equation

$$
\partial_{t} f+v \cdot \nabla_{x} f+E \cdot \nabla_{v} f+\operatorname{div}_{v}\left(\left(\alpha-\beta|v|^{2}\right) v f\right)=0,
$$

which is a linear first-order equation. The associated characteristic system of ode's is

$$
\begin{aligned}
& \frac{d}{d t} X=V, \\
& \frac{d}{d t} V=E(t, X)+V\left(\alpha-\beta|V|^{2}\right) .
\end{aligned}
$$

Lemma 3.2 (Flow Map). Take a field $E:[0, T] \times \mathbb{R}^{d} \rightarrow \mathbb{R}^{d}$ satisfying Hypothesis 3.1. Given $\left(X_{0}, V_{0}\right) \in \mathbb{R}^{d} \times \mathbb{R}^{d}$ there exists a unique solution $(X, V)$ to equations (11a)-(11b) in $\mathcal{C}^{1}\left([0, T] ; \mathbb{R}^{d} \times \mathbb{R}^{d}\right)$ satisfying $X(0)=X_{0}$ and $V(0)=V_{0}$. In addition, there exists a constant $C$ which depends only on $T,\left|X_{0}\right|,\left|V_{0}\right|, \alpha, \beta$ and the constant $C_{E}$ in eq. (8), such that

$$
|(X(t), V(t))| \leq\left|\left(X_{0}, V_{0}\right)\right| e^{C t} \quad \text { for all } t \in[0, T] .
$$

Proof. As the field $E$ satisfies the regularity and growth conditions in Hypothesis 3.1, standard results in ordinary differential equations show that for each initial condition $(X(0), V(0)) \in \mathbb{R}^{d} \times \mathbb{R}^{d}$ this system has a unique solution defined on $[0, T)$ (the only term in the equations which does not grow linearly is $-\beta V|V|^{2}$, and it makes $|V|$ decrease, so the solution is globally defined in time). The bound (12) on the solutions follows from direct estimates on the equation, using the linear growth of the field $E$. 
Calling $P \equiv(X, V)$, the system (11a)-(11b) can be conveniently written as

$$
\frac{d}{d t} P=\Psi_{E}(t, P)
$$

where $\Psi_{E}:[0, T] \times \mathbb{R}^{d} \times \mathbb{R}^{d} \rightarrow \mathbb{R}^{d} \times \mathbb{R}^{d}$ is the right hand side of eqs. (11a), (11b). When the field $E$ is understood we will just write $\Psi$ instead of $\Psi_{E}$. Using this notation, equation (10) can also be rewriten as

$$
\frac{\partial f}{\partial t}+\operatorname{div}\left(\Psi_{E} f\right)=0 .
$$

We can thus consider the flow at time $t \in[0, T)$ of eqs. (11),

$$
\mathcal{T}_{E}^{t}: \mathbb{R}^{d} \times \mathbb{R}^{d} \rightarrow \mathbb{R}^{d} \times \mathbb{R}^{d} .
$$

Again by basic results in ode's, the map $(t, x, v) \mapsto \mathcal{T}_{E}^{t}(x, v)=(X, V)$ with $(X, V)$ the solution at time $t$ to (12) with initial data $(x, v)$, is jointly continuous in $(t, x, v)$. For a measure $f_{0} \in \mathcal{P}_{1}\left(\mathbb{R}^{d} \times \mathbb{R}^{d}\right)$ it is well-known that the function

$$
f:[0, T) \rightarrow \mathcal{P}_{1}\left(\mathbb{R}^{d} \times \mathbb{R}^{d}\right), \quad t \mapsto f_{t}:=\mathcal{T}_{E}^{t} \# f_{0}
$$

is a measure solution to eq. (10), i.e., a solution in the distributional sense. Here we are using the mass transportation notation of push-forward: $f_{t}=\mathcal{T}_{E}^{t} \# f_{0}$ is defined by

$$
\int_{\mathbb{R}^{2 d}} \zeta(x, v) f(t, x, v) d(x, v)=\int_{\mathbb{R}^{2 d}} \zeta\left(\mathcal{T}_{E}^{t}(x, v)\right) f_{0}(x, v) d(x, v),
$$

for all $\zeta \in \mathcal{C}_{b}^{0}\left(\mathbb{R}^{2 d}\right)$. Note that in the case where the initial condition $f_{0}$ is regular $\left(\right.$ say, $\mathcal{C}_{c}^{\infty}$ ) this is just a way to rewrite the solution of the equation through the method of characteristics. This motivates the following definition:

Definition 3.3 (Notion of Solution). Take a potential $U \in \mathcal{C}^{1}\left(\mathbb{R}^{d}\right)$ such that

$$
|\nabla U(x)| \leq C(1+|x|), \quad x \in \mathbb{R}^{d},
$$

for some constant $C>0$. Take also a measure $f_{0} \in \mathcal{P}_{1}\left(\mathbb{R}^{d} \times \mathbb{R}^{d}\right)$, and $T \in$ $(0, \infty]$. We say that a function $f:[0, T] \rightarrow \mathcal{P}_{1}\left(\mathbb{R}^{d} \times \mathbb{R}^{d}\right)$ is a solution of the swarming equation (1) with initial condition $f_{0}$ when:

1. The field $E[f]=-\nabla U * \rho$ satisfies the conditions in Hypothesis 3.1.

2. It holds $f_{t}=\mathcal{T}_{E[f]}^{t} \# f_{0}$.

Remark 3.4. This definition gives a convenient condition on $U$ so that a measure solution in $\mathcal{P}_{1}\left(\mathbb{R}^{d} \times \mathbb{R}^{d}\right)$ makes sense. One can weaken the requirement on $U$ in this definition as long as the requirements on $f$ are suitably strengthened (e.g., one can allow a faster growth of the potential if one imposes a faster decay of $f$, or less local regularity of $U$ if one assumes more regularity of $f$ ), but we will not consider these modifications in the present paper. 
Since we ask the gradient of the potential to be locally Lipschitz, we cannot consider potentials with a singularity at the origin. This is a strong limitation of the classical theory, and is considered a difficult problem for the mean-field limit. As for the existence theory, if one wants to consider more singular potentials, one can work with functions $f$ which are more regular than just measures, so that $\nabla U * \rho$ becomes locally Lipschitz and a parallel existence theory can be developed.

\subsection{Estimates on the characteristics}

We gather in this section some estimates on solutions to the characteristic equations (11). In this section we fix $T>0$ and fields $E, E^{1}, E^{2}:[0, T] \times \mathbb{R}^{d} \rightarrow \mathbb{R}^{d}$ which are assumed to satisfy Hypothesis 3.1 and we consider their corresponding characteristic equations (11). Recall that $\Psi_{E}$ is a shorthand for the right hand side of (11), as in (13).

We first gather some basic regularity results for the function which defines the right hand side of eqs. (11a)-111b):

Lemma 3.5 (Regularity of the characteristic equations). Take a field $E$ : $[0, T] \times \mathbb{R}^{d} \rightarrow \mathbb{R}^{d}$ which satisfies Hypothesis 3.1. Consider a number $R>0$ and the closed ball $B_{R} \subseteq \mathbb{R}^{d} \times \mathbb{R}^{d}$.

1. $\Psi_{E}$ is bounded in compact sets: For $P=(X, V) \in B_{R}$ and $t \in[0, T]$,

$$
\left|\Psi_{E}(t, P)\right| \leq C
$$

for some $C>0$ which depends only on $\alpha, \beta, R$, and $\|E\|_{L^{\infty}\left([0, T] \times B_{R}\right)}$.

2. $\Psi_{E}$ is locally Lipschitz with respect to $x, v$ : For all $P_{1}=\left(X_{1}, V_{1}\right), P_{2}=$ $\left(X_{2}, V_{2}\right)$ in $B_{R}$, and $t \in[0, T]$,

$$
\left|\Psi_{E}\left(t, P_{1}\right)-\Psi_{E}\left(t, P_{2}\right)\right| \leq C\left(1+\operatorname{Lip}_{R}\left(E_{t}\right)\right)\left|P_{1}-P_{2}\right|,
$$

for some number $C>0$ which depends only on $\alpha$ and $\beta$.

Proof. This can be obtained by a direct calculation from eqs. (11a) (11b).

Lemma 3.6 (Dependence of the characteristic equations on $E$ ). Take two fields $E^{1}, E^{2}:[0, T] \times \mathbb{R}^{d} \rightarrow \mathbb{R}^{d}$ satisfying Hypothesis 3.1, and consider the functions $\Psi_{E^{1}}, \Psi_{E^{2}}$ which define the characteristic equations (11) as in eq. (13). Then, for any compact (in fact, any measurable) set B,

$$
\left\|\Psi_{E^{1}}-\Psi_{E^{2}}\right\|_{L^{\infty}(B)} \leq\left\|E^{1}-E^{2}\right\|_{L^{\infty}(B)} .
$$

Proof. Trivial from the expression of $\Psi_{E^{1}}, \Psi_{E^{2}}$.

Now we explicitly state some results which give a quantitative bound on the regularity of the flow $\mathcal{T}_{E}^{t}$, and its dependence on the field $E$. 
Lemma 3.7 (Dependence of characteristics on $E$ ). Take two fields $E^{1}, E^{2}$ : $[0, T] \times \mathbb{R}^{d} \rightarrow \mathbb{R}^{d}$ satisfying Hypothesis $\left[3.1\right.$, and a point $P^{0} \in \mathbb{R}^{d} \times \mathbb{R}^{d}$. Take $R>0$, and assume that

$$
\left|\mathcal{T}_{E^{1}}^{t}\left(P^{0}\right)\right| \leq R, \quad\left|\mathcal{T}_{E^{2}}^{t}\left(P^{0}\right)\right| \leq R \quad \text { for } t \in[0, T] .
$$

Then for $t \in[0, T]$ it holds that for some constant $C$ which depends only on $\alpha$, $\beta, R$ and $\operatorname{Lip}_{R}\left(E^{1}\right)$

$$
\left|\mathcal{T}_{E^{1}}^{t}\left(P^{0}\right)-\mathcal{T}_{E^{2}}^{t}\left(P^{0}\right)\right| \leq \frac{e^{C t}-1}{C} \sup _{s \in[0, T)}\left\|E_{s}^{1}-E_{s}^{2}\right\|_{L^{\infty}\left(B_{R}\right)} .
$$

Proof. For ease of notation, write $P_{i}(t) \equiv \mathcal{T}_{E_{i}}^{t}\left(P^{0}\right) \equiv\left(X_{i}(t), V_{i}(t)\right)$, for $i=1,2$, $t \in[0, T]$. These functions satisfy the characteristic equations (11):

$$
\frac{d}{d t} P_{i}=\Psi_{E_{i}}\left(t, P_{i}\right), \quad P_{i}(0)=P^{0}, \quad \text { for } i=1,2 .
$$

Then, for $t \in[0, T]$, and using Lemmas 3.5 and 3.6 .

$$
\begin{aligned}
\left|P_{1}(t)-P_{2}(t)\right| \leq & \int_{0}^{t}\left|\Psi_{E^{1}}\left(s, P_{1}(s)\right)-\Psi_{E^{2}}\left(s, P_{2}(s)\right)\right| d s \\
\leq & \int_{0}^{t}\left|\Psi_{E^{1}}\left(s, P_{1}(s)\right)-\Psi_{E^{1}}\left(s, P_{2}(s)\right)\right| d s \\
& +\int_{0}^{t}\left|\Psi_{E^{1}}\left(s, P_{2}(s)\right)-\Psi_{E^{2}}\left(s, P_{2}(s)\right)\right| d s \\
\leq & C \int_{0}^{t}\left|P_{1}(s)-P_{2}(s)\right| d s+\int_{0}^{t}\left\|E_{s}^{1}-E_{s}^{2}\right\|_{L^{\infty}\left(B_{R}\right)} d s
\end{aligned}
$$

where $C$ is the constant in point 2 of Lemma 3.5, which depends on $\alpha, \beta, R$ and the Lipschitz constant of $E^{1}$ with respect to $x$ in the ball $B_{R}$. By Gronwall's Lemma,

$$
\begin{aligned}
\left|P_{1}(t)-P_{2}(t)\right| & \leq \int_{0}^{t} e^{C(t-s)}\left\|E_{s}^{1}-E_{s}^{2}\right\|_{L^{\infty}\left(B_{R}\right)} d s . \\
& \leq \frac{e^{C t}-1}{C} \sup _{s \in(0, T)}\left\|E_{s}^{1}-E_{s}^{2}\right\|_{L^{\infty}\left(B_{R}\right)},
\end{aligned}
$$

which finishes the proof.

Lemma 3.8 (Regularity of characteristics with respect to initial conditions). Take $T>0$ and a field $E:[0, T] \times \mathbb{R}^{d} \rightarrow \mathbb{R}^{d}$ satisfying Hypothesis 3.1. Take also $P_{1}, P_{2} \in \mathbb{R}^{d} \times \mathbb{R}^{d}$ and $R>0$, and assume that

$$
\left|\mathcal{T}_{E}^{t}\left(P_{1}\right)\right| \leq R, \quad\left|\mathcal{T}_{E}^{t}\left(P_{2}\right)\right| \leq R \quad t \in[0, T]
$$

Then it holds that

$$
\left|\mathcal{T}_{E}^{t}\left(P_{1}\right)-\mathcal{T}_{E}^{t}\left(P_{2}\right)\right| \leq\left|P_{1}-P_{2}\right| e^{C \int_{0}^{t}\left(\operatorname{Lip}_{R}\left(E_{s}\right)+1\right) d s}, \quad t \in[0, T],
$$


for some constant $C$ which depends only on $R, \alpha$ and $\beta$. Said otherwise, $\mathcal{T}_{E}^{t}$ is Lipschitz on $B_{R} \subseteq \mathbb{R}^{d} \times \mathbb{R}^{d}$, with constant

$$
\operatorname{Lip}_{R}\left(\mathcal{T}_{E}^{t}\right) \leq e^{C \int_{0}^{t}\left(\operatorname{Lip}_{R}\left(E_{s}\right)+1\right) d s}, \quad t \in[0, T] .
$$

Proof. Write $P_{i}(t) \equiv \mathcal{T}_{E}^{t}\left(P_{i}\right) \equiv\left(X_{i}(t), V_{i}(t)\right)$, for $i=1,2, t \in[0, T]$. These functions satisfy the characteristic equations (11):

$$
\frac{d}{d t} P_{i}=\Psi_{E}\left(t, P_{i}\right), \quad P_{i}(0)=P_{i}, \quad \text { for } i=1,2 .
$$

For $t \in[0, T]$, using Lemma 3.5 .

$$
\begin{aligned}
\left|P_{1}(t)-P_{2}(t)\right| & \leq\left|P_{1}-P_{2}\right|+\int_{0}^{t}\left|\Psi_{E}\left(s, P_{1}(s)\right)-\Psi_{E}\left(s, P_{2}(s)\right)\right| d s \\
& \leq\left|P_{1}-P_{2}\right|+C \int_{0}^{t}\left(\operatorname{Lip}_{R}\left(E_{s}\right)+1\right)\left|P_{1}(s)-P_{2}(s)\right| d s
\end{aligned}
$$

We get our result by applying Gronwall's Lemma to this inequality.

Lemma 3.9 (Regularity of characteristics with respect to time). Take $T>0$ and a field $E:[0, T] \times \mathbb{R}^{d} \rightarrow \mathbb{R}^{d}$ satisfying Hypothesis 3.1. Take $P^{0} \in \mathbb{R}^{d} \times \mathbb{R}^{d}$, $R>0$ and assume that

$$
\left|\mathcal{T}_{E}^{t}\left(P^{0}\right)\right| \leq R, \quad t \in[0, T]
$$

Then it holds that

$$
\left|\mathcal{T}_{E}^{t}\left(P^{0}\right)-\mathcal{T}_{E}^{s}\left(P^{0}\right)\right| \leq C|t-s| \quad \text { for } s, t \in[0, T],
$$

for some constant $C$ which depends only on $\alpha, \beta, R$ and $\|E\|_{L^{\infty}\left([0, T] \times B_{R}\right)}$.

Proof. Direct by definition of $\mathcal{T}_{E}^{t}\left(P^{0}\right)$ and from point 1 of Lemma 3.5, as we are assuming that $\mathcal{T}_{E}^{t}\left(P^{0}\right)$ remains on a certain compact subset of $\mathbb{R}^{d} \times \mathbb{R}^{d}$.

\subsection{Existence and uniqueness}

Theorem 3.10 (Existence and uniqueness of measure solutions). Take a potential $U \in \mathcal{C}^{1}\left(\mathbb{R}^{d}\right)$ such that $\nabla U$ is locally Lipschitz and such that for some $C>0$,

$$
|\nabla U(x)| \leq C(1+|x|) \quad \text { for all } x \in \mathbb{R}^{d},
$$

and $f_{0} \in \mathcal{P}_{1}\left(\mathbb{R}^{d} \times \mathbb{R}^{d}\right)$ with compact support. There exists a solution $f$ on $[0,+\infty)$ to equation (11) with initial condition $f_{0}$ in the sense of Definition 3.3 . In addition,

$$
f \in \mathcal{C}\left([0,+\infty) ; \mathcal{P}_{1}\left(\mathbb{R}^{d} \times \mathbb{R}^{d}\right)\right)
$$

and there is some increasing function $R=R(T)$ such that for all $T>0$,

$$
\operatorname{supp} f_{t} \subseteq B_{R(T)} \subseteq \mathbb{R}^{d} \times \mathbb{R}^{d} \quad \text { for all } t \in[0, T] .
$$

This solution is unique among the family of solutions satisfying (21) and (22). 
The rest of this section is dedicated to the proof of this result, for which we will need some previous lemmas. We begin with a general result on the transportation of a measure by two different functions:

Lemma 3.11. Let $P_{1}, P_{2}: \mathbb{R}^{d} \rightarrow \mathbb{R}^{d}$ be two Borel measurable functions. Also, take $f \in \mathcal{P}_{1}\left(\mathbb{R}^{d}\right)$. Then,

$$
W_{1}\left(P_{1} \# f, P_{2} \# f\right) \leq\left\|P_{1}-P_{2}\right\|_{L^{\infty}(\operatorname{supp} f)} .
$$

Proof. We consider a transference plan defined by $\pi:=\left(P_{1} \times P_{2}\right) \# f$. One can check that this measure has marginals $P_{1} \# f, P_{2} \# f$. Then,

$$
\begin{aligned}
W_{1}\left(P_{1} \# f, P_{2} \# f\right) & \leq \int_{\mathbb{R}^{d} \times \mathbb{R}^{d}}|x-y| \pi(x, y) d x d y \\
& =\int_{\mathbb{R}^{d}}\left|P_{1}(x)-P_{2}(x)\right| f(x) d x \leq\left\|P_{1}-P_{2}\right\|_{L^{\infty}(\operatorname{supp} f)},
\end{aligned}
$$

which proves the lemma.

Lemma 3.12 (Continuity with respect to time). Take $T>0$ and a field $E$ : $[0, T] \times \mathbb{R}^{d} \rightarrow \mathbb{R}^{d}$ in the conditions of Hypothesis [3.1. Take also a measure $f$ on $\mathbb{R}^{d} \times \mathbb{R}^{d}$ with compact support contained in the ball $B_{R}$.

Then, there exists $C>0$ depending only on $\alpha, \beta, R$ and $\|E\|_{L^{\infty}\left([0, T] \times B_{R}\right)}$ such that

$$
W_{1}\left(\mathcal{T}_{E}^{s} \# f, \mathcal{T}_{E}^{t} \# f\right) \leq C|t-s|, \quad \text { for any } t, s \in[0, T] .
$$

Proof. From Lemma 3.11 and the continuity of characteristics with respect to time, Lemma 3.9. we get

$$
W_{1}\left(\mathcal{T}_{E}^{s} \# f, \mathcal{T}_{E}^{t} \# f\right) \leq\left\|\mathcal{T}_{E}^{s}-\mathcal{T}_{E}^{t}\right\|_{L^{\infty}(\operatorname{supp} f)} \leq C|t-s|,
$$

for some $C>0$ which depends only on the quantities in the lemma.

Lemma 3.13. Take a locally Lipschitz map $\mathcal{T}: \mathbb{R}^{d} \rightarrow \mathbb{R}^{d}$ and $f, g \in \mathcal{P}_{1}\left(\mathbb{R}^{d}\right)$, both with compact support contained in the ball $B_{R}$. Then,

$$
W_{1}(\mathcal{T} \# f, \mathcal{T} \# g) \leq L W_{1}(f, g),
$$

where $L$ is the Lipschitz constant of $\mathcal{T}$ on the ball $B_{R}$.

Proof. Set $\pi$ to be an optimal transportation plan between $f$ and $g$. The measure $\gamma=(\mathcal{T} \times \mathcal{T}) \# \pi$ has marginals $\mathcal{T} \# f$ and $\mathcal{T} \# g$, as can be easily checked, so we can use it to bound $W_{1}(\mathcal{T} \# f, \mathcal{T} \# g)$ :

$$
\begin{aligned}
W_{1}(\mathcal{T} \# f, \mathcal{T} \# g) & \leq \int_{\mathbb{R}^{d} \times \mathbb{R}^{d}}|z-w| \gamma(z, w) d z d w=\int_{\mathbb{R}^{d} \times \mathbb{R}^{d}}|\mathcal{T}(z)-\mathcal{T}(w)| \pi(z, w) d z d w \\
& \leq L \int_{\mathbb{R}^{d} \times \mathbb{R}^{d}}|z-w| \pi(z, w) d z d w=L W_{1}(f, g),
\end{aligned}
$$

using that the support of $\pi$ is contained in $B_{R} \times B_{R}$, as both $f$ and $g$ have support inside $B_{R}$. 
Recalling that $E[f]:=\nabla U * \rho$, the properties of convolution lead immediately to the following information:

Lemma 3.14. Take a potential $U: \mathbb{R}^{d} \rightarrow \mathbb{R}$ in the conditions of Theorem 3.10 , and a measure $f \in \mathcal{P}_{1}\left(\mathbb{R}^{d} \times \mathbb{R}^{d}\right)$ with support contained in a ball $B_{R}$. Then,

$$
\|E[f]\|_{L^{\infty}\left(B_{R}\right)} \leq\|\nabla U\|_{L^{\infty}\left(B_{2 R}\right)},
$$

and

$$
\operatorname{Lip}_{R}(E[f]) \leq \operatorname{Lip}_{2 R}(\nabla U) .
$$

Lemma 3.15. For $f, g \in \mathcal{P}_{1}\left(\mathbb{R}^{d} \times \mathbb{R}^{d}\right)$ and $R>0$ it holds that

$$
\|E[f]-E[g]\|_{L^{\infty}\left(B_{R}\right)} \leq \operatorname{Lip}_{2 R}(\nabla U) W_{1}(f, g) .
$$

Proof. Take $\pi$ to be an optimal transportation plan between the measures $f$ and $g$. Then, for any $x \in B_{R}$, using that $\pi$ has marginals $f$ and $g$,

$$
\begin{aligned}
E[f](x) & -E[g](x)=\int_{\mathbb{R}^{d}}(\rho[f](y)-\rho[g](y)) \nabla U(x-y) d y \\
= & \int_{\mathbb{R}^{d} \times \mathbb{R}^{d}} f(y, v) \nabla U(x-y) d y d v-\int_{\mathbb{R}^{d} \times \mathbb{R}^{d}} g(z, w) \nabla U(x-z) d z d w \\
= & \int_{\mathbb{R}^{4 d}}(\nabla U(x-y)-\nabla U(x-z)) d \pi(y, v, z, w) .
\end{aligned}
$$

Taking absolute value,

$$
\begin{aligned}
|E[f](z)-E[g](z)| & \leq \int_{\mathbb{R}^{4 d}}|\nabla U(x-y)-\nabla U(x-z)| d \pi(y, v, z, w) \\
& \leq \operatorname{Lip}_{2 R}(\nabla U) \int_{\mathbb{R}^{4 d}}|y-z| d \pi(y, v, z, w) \leq \operatorname{Lip}_{2 R}(\nabla U) W_{1}(f, g),
\end{aligned}
$$

using that $\pi(y, v, z, w)$ has support on $B_{R} \times B_{R} \subseteq \mathbb{R}^{4 d}$.

We can now give the proof of the existence and uniqueness result.

Proof of theorem 3.10. Take $f_{0} \in \mathcal{P}^{1}\left(\mathbb{R}^{d} \times \mathbb{R}^{d}\right)$ with support contained in a ball $B_{R^{0}} \subseteq \mathbb{R}^{d} \times \mathbb{R}^{d}$, for some $R^{0}>0$. We will prove local existence and uniqueness of solutions by a contraction argument in the metric space $\mathcal{F}$ formed by all the functions $f \in \mathcal{C}\left([0, T], \mathcal{P}_{1}\left(\mathbb{R}^{d} \times \mathbb{R}^{d}\right)\right)$ such that the support of $f_{t}$ is contained in $B_{R}$ for all $t \in[0, T]$, where $R:=2 R^{0}$ and $T>0$ is a fixed number to be chosen later. Here, we consider the distance in $\mathcal{F}$ given by

$$
\mathcal{W}_{1}(f, g):=\sup _{t \in[0, T]} W_{1}\left(f_{t}, g_{t}\right) \text {. }
$$

Let us define an operator on this space for which a fixed point will be a solution to the swarming equation (11). For $f \in \mathcal{F}$, consider $E[f]:=\nabla U * \rho[f]$. 
Then, $E[f]$ satisfies Hypothesis 3.1 (because of the above two Lemmas 3.14 and 3.15, and the bound (20) on $\nabla U$ ) and we can define

$$
\Gamma[f](t):=\mathcal{T}_{E[f]}^{t} \# f_{0} .
$$

In other words, $\Gamma[f]$ is the solution of the swarming equations obtained through the method of characteristics, with field $E[f]$ assumed known, and with initial condition $f_{0}$ at $t=0$.

Clearly, a fixed point of $\Gamma$ is a solution to eq. (11) on $[0, T]$. In order for $\Gamma$ to be well defined, we need to prove that $\Gamma[f]$ is again in the space $\mathcal{F}$, for which we need to choose $T$ appropriately. To do this, observe that from eq. (25) in Lemma 3.14 we have

$$
\|E[f]\|_{L^{\infty}\left([0, T] \times B_{R}\right)} \leq\|\nabla U\|_{L^{\infty}\left(B_{2 R}\right)}=: C_{1},
$$

and from point 1 in lemma 3.5

$$
\left|\frac{d}{d t} \mathcal{T}_{E[f]}^{t}(P)\right| \leq C_{2}
$$

for all $P \in B_{R^{0}} \subseteq \mathbb{R}^{d} \times \mathbb{R}^{d}$, and some $C_{2}>0$ which depends only on $\alpha, \beta, R_{0}$ and $C_{1}$. Choosing any $T<R^{0} / C_{2}$ one easily sees that $\mathcal{T}_{E[f]}^{t} \# f_{0}$ has support contained in $B_{R}$, for all $t \in[0, T]$ (recall that we set $R:=2 R^{0}$ ). Then, for each $t \in[0, T], \Gamma[f](t) \in \mathcal{P}_{1}\left(\mathbb{R}^{d} \times \mathbb{R}^{d}\right)$, as follows from mass conservation, the support of $\Gamma[f](t)$ is contained in $B_{R}$ (we just chose $T$ for this to hold), and the function $t \mapsto \Gamma[f](t)$ is continuous, as shown by Lemma 3.12. This shows that the map $\Gamma: \mathcal{F} \rightarrow \mathcal{F}$ is well defined.

Let us prove now that this map is contractive (for which we will have to restrict again the choice of $T$ ). Take two functions $f, g \in \mathcal{F}$, and consider $\Gamma[f], \Gamma[g]$; we want to show that

$$
\mathcal{W}_{1}(\Gamma[f], \Gamma[g]) \leq C \mathcal{W}_{1}(f, g)
$$

for some $0<C<1$ which does not depend on $f$ and $g$. Using (28) and (29),

$$
\mathcal{W}_{1}(\Gamma[f], \Gamma[g])=\sup _{t \in[0, T]} W_{1}\left(\mathcal{T}_{E[f]}^{t} \# f_{0}, \mathcal{T}_{E[g]}^{t} \# f_{0}\right),
$$

and hence we need to estimate the above quantity for each $t \in[0, T)$. For $t \in[0, T]$, use lemmas 3.11, 3.7 and 3.15 to write

$$
\begin{aligned}
W_{1}\left(\mathcal{T}_{E[f]}^{t} \# f_{0}, \mathcal{T}_{E[g]}^{t} \# f_{0}\right) & \leq\left\|\mathcal{T}_{E[f]}^{t}-\mathcal{T}_{E[g]}^{t}\right\|_{L^{\infty}\left(\operatorname{supp} f_{0}\right)} \\
& \leq C(t) \sup _{s \in[0, T]}\left\|E\left[f_{s}\right]-E\left[g_{s}\right]\right\|_{L^{\infty}\left(B_{R}\right)} \\
& \leq C(t) L \sup _{s \in[0, T]} W_{1}\left(f_{s}, g_{s}\right)=C(t) L \mathcal{W}_{1}(f, g)
\end{aligned}
$$


where $C(t)$ is the function $\left(e^{C_{3} t}-1\right) / C_{3}$ which appears in eq. (17), for some constant $C_{3}$ which depends only on $\alpha, \beta, R$, and the Lipschitz constant $L$ of $\nabla U$ on $B_{2 R}$ (see eq. (26)). Clearly,

$$
\lim _{t \rightarrow 0} C(t)=0
$$

With (31), this finally gives

$$
\mathcal{W}_{1}(\Gamma[f], \Gamma[g]) \leq C(T) L \mathcal{W}_{1}(f, g) .
$$

Taking into account (32), we can additionally choose $T$ small enough so that $C(T) L<1$. For such $T, \Gamma$ is contractive, and this proves that there is a unique fixed point of $\Gamma$ in $\mathcal{F}$, and hence a unique solution $f \in \mathcal{F}$ of eq. (11).

Finally, as mass is conserved, by usual arguments one can extend this solution as long as the support of the solution remains compact. Since in our case the growth of characteristics is bounded (see Lemma 3.2), one can construct a unique global solution satisfying (21) and (22).

\subsection{Stability}

Theorem 3.16. Take a potential $U$ in the conditions of Theorem 3.10, and $f_{0}$, $g_{0}$ measures on $\mathbb{R}^{d} \times \mathbb{R}^{d}$ with compact support, and consider the solutions $f, g$ to eq. (11) given by Theorem [3.10 with initial data $f_{0}$ and $g_{0}$, respectively.

Then, there exists a strictly increasing smooth function $r(t):[0, \infty) \longrightarrow \mathbb{R}_{0}^{+}$ with $r(0)=1$ depending only on the size of the support of $f_{0}$ and $g_{0}$, such that

$$
W_{1}\left(f_{t}, g_{t}\right) \leq r(t) W_{1}\left(f_{0}, g_{0}\right), \quad t \geq 0 .
$$

Proof. Fix $T>0$, and take $R>0$ such that $\operatorname{supp} f_{t}$ and $\operatorname{supp} g_{t}$ are contained in $B_{R}$ for $t \in[0, T]$ (which can be done thanks to theorem [3.10). For $t \in[0, T]$, call $L_{t}$ the Lipschitz constant of $\mathcal{T}_{E[g]}^{t}$ on $B_{R}$, and notice that from lemmas 3.8 and 3.14 we have

$$
L_{t} \leq e^{C_{1} t}, \quad t \in[0, T]
$$

for some allowed constant $C_{1}>0$. Then we have, using lemmas 3.11 , 3.13, 3.7 and 3.15

$$
\begin{aligned}
W_{1}\left(f_{t}, g_{t}\right) & =W_{1}\left(\mathcal{T}_{E[f]}^{t} \# f_{0}, \mathcal{T}_{E[g]}^{t} \# g_{0}\right) \\
& \leq W_{1}\left(\mathcal{T}_{E[f]}^{t} \# f_{0}, \mathcal{T}_{E[g]}^{t} \# f_{0}\right)+W_{1}\left(\mathcal{T}_{E[g]}^{t} \# f_{0}, \mathcal{T}_{E[g]}^{t} \# g_{0}\right) \\
& \leq\left\|\mathcal{T}_{E[f]}^{t}-\mathcal{T}_{E[g]}^{t}\right\|_{L^{\infty}\left(\operatorname{supp} f_{0}\right)}+L_{t} W_{1}\left(f_{0}, g_{0}\right) \\
& \leq C_{2} \int_{0}^{t} e^{C_{2}(t-s)}\left\|E\left[f_{s}\right]-E\left[g_{s}\right]\right\|_{L^{\infty}\left(B_{R}\right)} d s+L_{t} W_{1}\left(f_{0}, g_{0}\right) \\
& \leq C_{2} \operatorname{Lip}_{2 R}(\nabla U) \int_{0}^{t} e^{C_{2}(t-s)} W_{1}\left(f_{s}, g_{s}\right) d s+e^{C_{1} t} W_{1}\left(f_{0}, g_{0}\right) .
\end{aligned}
$$


Calling $C=\max \left\{C_{1}, C_{2}, C_{2} \operatorname{Lip}_{2 R}(\nabla U)\right\}$ and multiplying by $e^{-C t}$,

$$
e^{-C t} W_{1}\left(f_{t}, g_{t}\right) \leq C \int_{0}^{t} e^{-C s} W_{1}\left(f_{s}, g_{s}\right) d s+W_{1}\left(f_{0}, g_{0}\right), \quad t \in[0, T],
$$

and then by Gronwall's Lemma,

$$
e^{-C t} W_{1}\left(f_{t}, g_{t}\right) \leq W_{1}\left(f_{0}, g_{0}\right) e^{C t}, \quad t \in[0, T]
$$

which proves our result. We point out that the particular rate function $r(t)$ can be obtained by carefully looking at the dependencies on time of the constants above, leading to double exponentials.

Remark 3.17 (Possible generalizations). As in Remark 3.4, by assuming more restrictive growth properties at infinity of the potential $U$, we may weaken the requirements on the support of the initial data allowing $f_{0}$ with bounded first moment for instance. We do not follow this strategy in the present work.

\subsection{Regularity}

If the initial condition for eq. (11) is more regular than a general measure on $\mathbb{R}^{d} \times \mathbb{R}^{d}$ one can easily prove that the solution $f$ is also more regular. For example, if $f_{0}$ is Lipschitz, then $f_{t}$ is Lipschitz for all $t \geq 0$. We will show this next.

Lemma 3.18. Take an integrable function $f_{0}: \mathbb{R}^{d} \times \mathbb{R}^{d} \rightarrow[0,+\infty)$, with compact support, and assume that $f_{0}$ is also Lipschitz. Take also a potential $U \in \mathcal{C}^{2}\left(\mathbb{R}^{D}\right)$.

Consider the global solution $f$ to eq. (11) with initial condition $f_{0}$ given by Theorem 3.10. Then, $f_{t}$ is Lipschitz for all $t \geq 0$.

Proof. Solutions obtained from Theorem 3.10 have bounded support in velocity for all times $t>0$, and their fields $E \equiv E(t, x):=-\nabla U * \rho$ are Lipschitz with respect to $x$. Hence, one can rewrite eq. (11) as a general equation of the form

$$
\partial_{t} f+\operatorname{div}(a f)=0
$$

where $a=a(t, x, v)$ is the expression appearing in the equation,

$$
a(t, x, v)=\left(v, E(t, x)+\left(\alpha-\beta|v|^{2}\right) v\right) .
$$

Then, $a$ is bounded and Lipschitz with respect to $x, v$ on the domain considered as the support in velocity is bounded, and classical results show that $f_{t}$ is Lipschitz for all $t \geq 0$.

\section{Well-posedness for General Models}

In this section we want to show that the same results we have obtained in the previous section are also valid, with suitable modifications, for much more general models than (11). We will start by showing the adaptation of the strategy for the Cucker-Smale system and then, we will extend this strategy to more general models. 


\subsection{Cucker-Smale Model}

We will prove well-posedness in a slightly more general setting than that of the Cucker-Smale model in section 2 being less restrictive on the communication rate and the velocity averaging. To be more precise, we shall consider $\xi[f](x, v, t)=[(H(x, v)) * f](x, v, t)$ as in (3), but for a general $H: \mathbb{R}^{d} \times \mathbb{R}^{d} \rightarrow$ $\mathbb{R}^{d}$, for which we only assume the following hypotheses:

Hypothesis 4.1 (Conditions on $H$ ). $\quad$ 1. $H$ is locally Lipschitz.

2. For some $C>0$,

$$
|H(x, v)| \leq C(1+|x|+|v|) \quad \text { for all } x, v \in \mathbb{R}^{d} .
$$

Since the procedure to prove the well-posedness results to (3) is the same we have already applied in the previous section, we will state some of the results without proof. First of all, fix $T>0$ and let us introduce the system of ODE's solved by the characteristics of (3):

$$
\begin{aligned}
& \frac{d}{d t} X=V, \\
& \frac{d}{d t} V=-\xi(t, X, V),
\end{aligned}
$$

where $\xi:[0, T] \times \mathbb{R}^{d} \times \mathbb{R}^{d} \rightarrow \mathbb{R}^{d}$ is any function satisfying the following hypothesis:

Hypothesis 4.2 (Conditions on $\xi$ ). $\quad$ 1. $\xi$ is continuous on $[0, T] \times \mathbb{R}^{d} \times \mathbb{R}^{d}$,

2. For some $C>0$,

$$
|\xi(t, x, v)| \leq C(1+|x|+|v|), \quad \text { for all } t, x, v \in[0, T] \times \mathbb{R}^{d} \times \mathbb{R}^{v} \text {, and }
$$

3. $\xi$ is locally Lipschitz with respect to $x$ and $v$, i.e., for any compact set $K \subseteq \mathbb{R}^{d} \times \mathbb{R}^{d}$ there is some $L_{K}>0$ such that

$$
\left|\xi\left(t, P_{1}\right)-\xi\left(t, P_{2}\right)\right| \leq L_{K}\left|P_{1}-P_{2}\right|, \quad t \in[0, T], \quad P_{1}, P_{2} \in K .
$$

Under these conditions, we may consider the flow map $P_{\xi}^{t}=P_{\xi}^{t}(x, v)$ associated to (36), defined as the solution to the system (36) with initial condition $(x, v)$. For ease of notation, we will write the system (36) as

$$
\frac{d P_{\xi}^{t}}{d t}=\Psi_{\xi}\left(t, P_{\xi}^{t}\right)
$$

Remark 4.3. Under Hypothesis 4.1 on $H$, note that whenever $\tilde{f} \in C\left([0, T], \mathcal{P}_{1}\left(\mathbb{R}^{d} \times\right.\right.$ $\left.\left.\mathbb{R}^{d}\right)\right)$ is a given compactly supported measure with $\operatorname{supp}\left(\tilde{f}_{t}\right) \subset B_{R^{x}} \times B_{R^{v}}$ for all $t \in[0, T]$, the field $\xi[\tilde{f}]=H * \tilde{f}$ satisfies Hypothesis [4.2. 
Definition 4.4 (Notion of Solution). Take $H$ satisfying Hypothesis 4.1, a measure $f_{0} \in \mathcal{P}_{1}\left(\mathbb{R}^{d} \times \mathbb{R}^{d}\right)$, and $T \in(0, \infty]$. We say that a function $f:[0, T] \rightarrow$ $\mathcal{P}_{1}\left(\mathbb{R}^{d} \times \mathbb{R}^{d}\right)$ is a solution of the swarming equation 3 with initial condition $f_{0}$ when:

1. The field $\xi=H * f$ satisfies Hypothesis 4.2.

2. It holds $f_{t}=P_{\xi}^{t} \# f_{0}$.

Now, an analogue to Lemma 3.5 can be stated. We shall state this one and the following lemmas for a general $\xi$ satisfying Hypothesis (4.2).

Lemma 4.5 (Regularity of the characteristic equations). Take $T>0$, $\xi$ satisfying Hypothesis (4.2), $R>0$ and $t \in[0, T]$. Then there exist constants $C$ and $L_{p}$ depending on $\operatorname{Lip}_{R}(\xi)$ and $T$ such that

$$
\left|\Psi_{\xi}(P)\right| \leq C \quad \text { for all } P \in B_{R} \times B_{R}
$$

and

$$
\left|\Psi_{\xi}\left(P_{1}\right)-\Psi_{\xi}\left(P_{2}\right)\right| \leq L_{p}\left|P_{1}-P_{2}\right| \quad \text { for all } P_{1}, P_{2} \in B_{R} \times B_{R} .
$$

Lemmas 3.6 3.9 are valid as they are presented, taking $\xi$ and Hypothesis 4.2 to play the role of $E$ and Hypothesis 3.1. and making the obvious minor modifications on the dependence of the constants. Now we can look at the existence of solutions:

Theorem 4.6 (Existence and uniqueness of measure solutions). Assume $H$ satisfies Hypothesis 4.1. and take $f_{0} \in \mathcal{P}_{1}\left(\mathbb{R}^{d} \times \mathbb{R}^{d}\right)$ compactly supported. Then there exists a unique solution $f \in C\left([0, T], \mathcal{P}_{1}\left(\mathbb{R}^{d} \times \mathbb{R}^{d}\right)\right)$ to equation (3) in the sense of Definition 4.4 with initial condition $f_{0}$. Moreover, the solution remains compactly supported for all $t \in[0, T]$, i.e., there exist $R^{x}$ and $R^{v}$ depending on $T, H$ and the support of $f_{0}$, such that

$$
\operatorname{supp}\left(f_{t}\right) \subset B_{R^{x}} \times B_{R^{v}} \text { for all } t \in[0, T] .
$$

The proof of this result can be done following the same steps as for proving Theorem 3.10, Lemmas 3.11 to 3.13 still hold in this situation, and we recombine Lemmas 3.14 and 3.15 in the following result:

Lemma 4.7. Take H satisfying Hypothesis 4.1. $\tilde{f} \in \mathcal{P}_{1}\left(\mathbb{R}^{d} \times \mathbb{R}^{d}\right)$ with $\operatorname{supp}\left(\tilde{f}_{t}\right) \subset$ $B_{R^{x}} \times B_{R^{v}}$, and $\xi:=\xi[\tilde{f}]=H * \tilde{f}$. Then, for any $R>0$

$$
\operatorname{Lip}_{R}(\xi) \leq \operatorname{Lip}_{R+\hat{R}}(H),
$$

with $\hat{R}:=\max R^{x}, R^{v}$. Furthermore, if $\tilde{g} \in \mathcal{P}_{1}\left(\mathbb{R}^{d} \times \mathbb{R}^{d}\right)$ it holds that

$$
\|\xi[\tilde{f}]-\xi[\tilde{g}]\|_{L^{\infty}\left(B_{R}\right)} \leq \operatorname{Lip}_{R+\hat{R}}(H) W_{1}(\tilde{f}, \tilde{g})
$$


Proof. The first part follows directly from the properties of convolution. For the second one, take $\pi$ to be an optimal transportation plan between the measures $\tilde{f}$ and $\tilde{g}$. Then, for any $x, v \in B_{R}$, using that $\pi$ has marginals $\tilde{f}$ and $\tilde{g}$,

$$
\begin{aligned}
\xi[\tilde{f}](x, v) & -\xi[\tilde{g}](x, v) \\
= & \int_{\mathbb{R}^{2 d}} H(x-y, v-u) \tilde{f}(y, u) d(y, u) \\
& -\int_{\mathbb{R}^{2 d}} H(x-z, v-w) \tilde{g}(z, w) d(z, w) \\
= & \int_{\mathbb{R}^{4 d}}[H(x-y, v-u)-H(x-z, v-w)] d \pi(y, u, z, w) .
\end{aligned}
$$

Taking absolute value, and using that the support of $\pi$ is contained in the ball $B_{\hat{R}} \subseteq \mathbb{R}^{4 d}$,

$$
\begin{aligned}
& \mid \xi[\tilde{f}](x, v)-\xi[\tilde{g}](x, v) \mid \\
& \leq \int_{B_{\hat{R}}}|H(x-y, v-u)-H(x-z, v-w)| d \pi(y, u, z, w) \\
& \leq \operatorname{Lip}_{R+\hat{R}}(H) \int_{\mathbb{R}^{4 n}}|(y-z, u-w)| d \pi(y, u, z, w)=\operatorname{Lip}_{R+\hat{R}}(H) W_{1}(\tilde{f}, \tilde{g}) .
\end{aligned}
$$

Finally, a stability result also follows using the same steps as in Theorem 3.16 .

Theorem 4.8 (Stability in $W_{1}$ ). Assume $H$ satisfies Hypothesis 4.1 , and $f_{0}, g_{0} \in$ $\mathcal{P}_{1}\left(\mathbb{R}^{d} \times \mathbb{R}^{d}\right)$ are compactly supported. Consider the solutions $f, g$ to eq. (3) given by Theorem 4.6 with initial data $f_{0}$ and $g_{0}$, respectively. Then, there exists a strictly increasing function $r(t):[0, \infty) \longrightarrow \mathbb{R}_{0}^{+}$with $r(0)=1$ depending only on $H$ and the size of the support of $f_{0}$ and $g_{0}$, such that

$$
W_{1}\left(f_{t}, g_{t}\right) \leq r(t) W_{1}\left(f_{0}, g_{0}\right), \quad t \geq 0 .
$$

Remark 4.9 (Evolution of the support in the Cucker-Smale model). In [8] it is shown a sharp bound on the evolution of the support for the kinetic CuckerSmale equation in which $H(x, v)=w(x) v$. More precisely, it is proved that for any given $f_{0} \in \mathcal{P}_{1}\left(\mathbb{R}^{d} \times \mathbb{R}^{d}\right)$ compactly supported, we have that

$$
\operatorname{supp}\left(f_{t}\right) \subset B\left(x_{c}(0)+m t, R^{x}(t)\right) \times B\left(m, R^{v}(t)\right),
$$

with

$$
R^{x}(t) \leq \bar{R} \quad \text { and } \quad R^{v}(t) \leq R_{0} e^{-\lambda t}
$$

for some $\bar{R}$ depending only on $R_{0}=\max \left\{R^{x}(0), R^{v}(0)\right\}$ and $\lambda=w(2 \bar{R})$. Here, $m$ stands for the mean velocity of the system

$$
m:=\int_{\mathbb{R}^{2 d}} v f(t, x, v) d x d v
$$


which is preserved along its evolution. This precise bound on the support and the particular choice of $H$ lead to a uniform control in time of the constants $\operatorname{Lip}_{R}(H)$ and $L_{p}$ in the results above, which are now bounded for all times. A tedious but straightforward computation leads to a rate $r(t)$ in the stability result which is exponentially increasing. Indeed, if we follow the steps of the proof of Theorem 3.16 for the particular case of the Cucker-Smale model we can see that, since $R_{x}$ and $R_{v}$ are not increasing with time, the numbers $C_{1}$ and $C_{2}$ that appear there can be chosen independently of time, whence $r(t)$ shall grow at most exponentially.

Remark 4.10 (Comparison with Literature). As already mentioned above, the particular case of the kinetic Cucker-Smale model has already been approached in [19] where the authors give a well-posedness result based on the bounded Lipschitz distance. Here, we recover the same result but based on the stability in the Wasserstein distance $W_{1}$, which allows us to obtain sharper constants and rates.

\subsection{General Models}

With the techniques used in the previous sections one can include quite general kinetic models in the well-posedness theory. In this section we illustrate this by giving a result for a model which includes both the potential interaction and self-propulsion effects of section 3, the velocity-averaging effect of section 4.1 and the more general models above 24, 25 .

Let us introduce some notation for this section: $\mathcal{P}_{c}\left(\mathbb{R}^{d} \times \mathbb{R}^{d}\right)$ denotes the subset of $\mathcal{P}_{1}\left(\mathbb{R}^{d} \times \mathbb{R}^{d}\right)$ consisting of measures of compact support in $\mathbb{R}^{d} \times \mathbb{R}^{d}$, and we consider the non-complete metric space $\mathcal{A}:=\mathcal{C}\left([0, T], \mathcal{P}_{c}\left(\mathbb{R}^{d} \times \mathbb{R}^{d}\right)\right)$ endowed with the distance $\mathcal{W}_{1}$. On the other hand, we consider the set of functions $\mathcal{B}:=\mathcal{C}\left([0, T], \operatorname{Lip}_{l o c}\left(\mathbb{R}^{d} \times \mathbb{R}^{d}, \mathbb{R}^{d}\right)\right)$, which in particular are locally Lipschitz with respect to $(x, v)$, uniformly in time. We consider an operator $\mathcal{H}[\cdot]: \mathcal{A} \longrightarrow \mathcal{B}$ and assume the following:

Hypothesis 4.11 (Hypothesis on a general operator). Take any $R_{0}>0$ and $f, g \in \mathcal{A}$ such that $\operatorname{supp}\left(f_{t}\right) \cup \operatorname{supp}\left(g_{t}\right) \subseteq B_{R_{0}}$ for all $t \in[0, T]$. Then for any ball $B_{R} \subset \mathbb{R}^{d} \times \mathbb{R}^{d}$, there exists a constant $C=C\left(R, R_{0}\right)$ such that

$$
\begin{aligned}
\max _{t \in[0, T]}\|\mathcal{H}[f]-\mathcal{H}[g]\|_{L^{\infty}\left(B_{R}\right)} & \leq C \mathcal{W}_{1}(f, g), \\
\max _{t \in[0, T]} \operatorname{Lip}_{R}(\mathcal{H}[f]) & \leq C .
\end{aligned}
$$

Associated to this operator, we can consider the following general equation:

$$
\partial_{t} f+v \cdot \nabla_{x} f-\nabla_{v} \cdot[\mathcal{H}[f] f]=0 .
$$

Remark 4.12 (Generalization). It is not difficult to see that the choices $\mathcal{H}[f]=$ $\left(\alpha-\beta|v|^{2}\right) v-\nabla U * \rho$ and $\mathcal{H}[f]=H * f$ correspond to (11) and (3), respectively, and that they satisfy Hypothesis 4.11 if we assume the hypotheses of Theorems 3.10 and 4.6 respectively. Moreover, one can cook up an operator of the form:

$$
\mathcal{H}[f]=F_{A}(x, v)+G(x) * \rho+H(x, v) * f
$$


with $F_{A}, G$ and $H$ given functions satisfying suitable hypotheses, such that the kinetic equation (41) corresponds to the model (4).

We will additionally require the following:

Hypothesis 4.13 (Additional constraint on $\mathrm{H})$. Given $f \in \mathcal{C}\left([0, T], \mathcal{P}_{c}\left(B_{R_{0}}\right)\right.$ ), and for any initial condition $\left(X^{0}, V^{0}\right) \in \mathbb{R}^{d} \times \mathbb{R}^{d}$, the following system of ordinary differential equations has a globally defined solution:

$$
\begin{aligned}
\frac{d}{d t} X & =V, \\
\frac{d}{d t} V & =\mathcal{H}[f](t, X, V), \\
X(0) & =X^{0}, \quad V(0)=V^{0} .
\end{aligned}
$$

Of course, this is a requirement that has to be checked for every particular model, and it is difficult to give useful properties of $\mathcal{H}$ that imply this and are general enough to encompass a range of utile models; therefore, we prefer to give a general condition which reduces the problem of existence and stability to the simpler one of existence of the characteristics.

In the above conditions one can follow a completely analogous argument to that in the proof of Theorems 3.10 and 3.16, and obtain the following result:

Theorem 4.14 (Existence, uniqueness and stability of measure solutions for a general model). Take an operator $\mathcal{H}[\cdot]: \mathcal{A} \longrightarrow \mathcal{B}$ satisfying Hypotheses 4.11 and 4.13 , and $f_{0}$ a measure on $\mathbb{R}^{d} \times \mathbb{R}^{d}$ with compact support. There exists a solution $f$ on $[0,+\infty)$ to equation (41) with initial condition $f_{0}$. In addition,

$$
f \in \mathcal{C}\left([0,+\infty) ; \mathcal{P}_{c}\left(\mathbb{R}^{d} \times \mathbb{R}^{d}\right)\right)
$$

and there is some increasing function $R=R(T)$ such that for all $T>0$,

$$
\operatorname{supp} f_{t} \subseteq B_{R(T)} \subseteq \mathbb{R}^{d} \times \mathbb{R}^{d} \quad \text { for all } t \in[0, T] .
$$

This solution is unique among the family of solutions satisfying (43) and (44).

Moreover, given any other initial data $g_{0} \in \mathcal{P}_{c}\left(\mathbb{R}^{d} \times \mathbb{R}^{d}\right)$ and $g$ its corresponding solution, then there exists a strictly increasing function $r(t):[0, \infty) \longrightarrow \mathbb{R}_{0}^{+}$ with $r(0)=1$ depending only on $\mathcal{H}$ and the size of the support of $f_{0}$ and $g_{0}$, such that

$$
W_{1}\left(f_{t}, g_{t}\right) \leq r(t) W_{1}\left(f_{0}, g_{0}\right), \quad t \geq 0 .
$$

\section{Consequences of Stability}

\subsection{N-Particle approximation and the mean-field limit}

The stability theorems 3.16 and 4.8, or the general version 4.14 give in particular a justification of the approximation of this family of models by a finite set of particles satisfying a system of ordinary differential equations. We will state results for the general model (41), under the conditions on $\mathcal{H}$ from section 4.2

One can easily check that the following holds: 
Lemma 5.1 (Particle solutions). Assume $\mathcal{H}$ satisfies the conditions of Theorem 4.14. Take $N$ positive numbers $m_{1}, \ldots, m_{N}$, and consider the following system of differential equations:

$$
\begin{array}{ll}
\dot{x_{i}}=v_{i}, & i=1, \ldots, N, \\
\dot{v}_{i}=\sum_{j \neq i} m_{j} \mathcal{H}\left[f^{N}\right]\left(t, x_{i}, v_{i}\right), & i=1, \ldots, N,
\end{array}
$$

where $f^{N}:[0, T] \rightarrow \mathcal{P}_{1}\left(\mathbb{R}^{d} \times \mathbb{R}^{d}\right)$ is the measure defined by

$$
f_{t}^{N}:=\sum_{i=1}^{N} m_{i} \delta_{\left(x_{i}(t), v_{i}(t)\right)} .
$$

If $x_{i}, v_{i}:[0, T] \rightarrow \mathbb{R}^{d}$, for $i=1, \ldots, N$, is a solution to the system (45), then the function $f^{N}$ is the solution to (41) with initial condition

$$
f_{0}^{N}=\sum_{i=1}^{N} m_{i} \delta_{\left(x_{i}(0), v_{i}(0)\right)} .
$$

As a consequence of the stability in $W_{1}$, we have an alternative method to derive the kinetic equations (11), (3) or (41), based on the convergence of particle approximations, other than the formal BBGKY hierarchy in [3, 7.

Corollary 5.2 (Convergence of the particle method). Given $f_{0} \in \mathcal{P}_{1}\left(\mathbb{R}^{d} \times \mathbb{R}^{d}\right)$ compactly supported and $\mathcal{H}$ satisfying the conditions of Theorem 4.14, take a sequence of $f_{0}^{N}$ of measures of the form (47) (with $m_{i}, x_{i}(0)$ and $v_{i}(0)$ possibly varying with $N$ ), in such a way that

$$
\lim _{N \rightarrow \infty} W_{1}\left(f_{0}^{N}, f_{0}\right)=0 .
$$

Consider $f_{t}^{N}$ given by (46), where $x_{i}(t)$ and $v_{i}(t)$ are the solution to system (45) with initial conditions $x_{i}(0), v_{i}(0)$. Then,

$$
\lim _{N \rightarrow \infty} W_{1}\left(f_{t}^{N}, f_{t}\right)=0
$$

for all $t \geq 0$, where $f=f(t, x, v)$ is the unique measure solution to eq. (41) with initial data $f_{0}$.

\section{$5.2 \quad$ Hydrodynamic limit}

We state our hydrodynamic limit result for eq. (1). If we look for solutions of (11) of the form

$$
f(t, x, v)=\rho(t, x) \delta(v-u(t, x))
$$

for some functions $\rho, u:[0, T] \times \mathbb{R}^{d} \rightarrow \mathbb{R}$, one formally obtains that $\rho$ and $u$ should satisfy the following equations:

$$
\begin{aligned}
& \partial_{t} \rho+\operatorname{div}_{x}(\rho u)=0, \\
& \partial_{t} u+(u \cdot \nabla) u=u\left(\alpha-\beta|u|^{2}\right)-\nabla U * \rho .
\end{aligned}
$$


This is made precise by the following result whose existence part was already obtained in [7:

Lemma 5.3 (Uniqueness for Hydrodynamic Solutions). Take a potential $U \in$ $\mathcal{C}^{2}\left(\mathbb{R}^{d}\right)$ and assume that there exists a smooth solution $(\rho, u)$ with initial data $\left(\rho_{0}, u_{0}\right)$ to the system (49) defined on the interval $[0, T]$. Then, if we define $f:[0,+\infty) \rightarrow \mathcal{P}_{1}\left(\mathbb{R}^{d} \times \mathbb{R}^{d}\right)$ by

$$
\int_{\mathbb{R}^{d} \times \mathbb{R}^{d}} f(t, x, v) \phi(x, v) d x d v=\int_{\mathbb{R}^{d}} \phi(x, u(t, x)) \rho(t, x) d x
$$

for any test function $\phi \in \mathcal{C}_{C}^{0}\left(\mathbb{R}^{d} \times \mathbb{R}^{d}\right)$, then $f$ is the unique solution to (1) obtained from Theorem 3.10 with initial condition $f_{0}=\rho_{0} \delta\left(v-u_{0}\right)$.

As a direct consequence of Lemma 5.3 and the stability result in Theorem 3.16, we get the following result.

Corollary 5.4 (Local-in-time Stability of Hydrodynamics). Take a potential $U \in \mathcal{C}^{2}\left(\mathbb{R}^{d}\right)$ and assume that there exists a smooth solution $(\rho, u)$ with initial data $\left(\rho_{0}, u_{0}\right)$ to the system (49) defined on the interval $[0, T]$. Let us consider a sequence of initial data $f_{0}^{k} \in \mathcal{P}_{1}\left(\mathbb{R}^{d} \times \mathbb{R}^{d}\right)$ such that

$$
\lim _{k \rightarrow \infty} W_{1}\left(f_{0}^{k}, \rho_{0} \delta\left(v-u_{0}\right)\right)=0 .
$$

Consider the solution $f^{k}$ to the swarming eq. (1) with initial data $f_{0}^{k}$. Then,

$$
\lim _{k \rightarrow \infty} W_{1}\left(f_{t}^{k}, f_{t}\right)=0
$$

for all $t \in[0, T]$ with $f(t, x, v)=\rho(t, x) \delta(v-u(t, x))$.

\section{Acknowledgments}

The authors acknowledge support from the project MTM2008-06349-C03-03 DGI-MCI (Spain) and 2009-SGR-345 from AGAUR-Generalitat de Catalunya. This work was developed at the CRM-Barcelona during the thematic program in "Mathematical Biology" in 2009.

\section{References}

[1] A. Barbaro, K. Taylor, P. F. Trethewey, L. Youseff and B. Birnir, Discrete and continuous models of the dynamics of pelagic fish: application to the capelin, preprint.

[2] B. Birnir, An ODE model of the motion of pelagic fish, J. Stat. Phys. 128 (2007) 535-568.

[3] N. N. Bogoliubov, Problems of a dynamical theory in statistical physics, Studies in Statistical Mechanics, Vol. I, (Interscience, New York, 1962). 
[4] W. Braun and K. Hepp, The Vlasov Dynamics and Its Fluctuations in the 1/N Limit of Interacting Classical Particles, Commun. Math. Phys. 56 (1977) 101-113.

[5] E. Bonabeau, M. Dorigo and G. Theraulaz, Swarm Intelligence: From Natural to Artificial Systems, (Oxford University Press, New York, 1999).

[6] S. Camazine, J.-L. Deneubourg, N.R. Franks, J. Sneyd, G. Theraulaz and E. Bonabeau, Self-Organization in Biological Systems, (Princeton University Press, 2003).

[7] J. A. Carrillo, M. R. D'Orsogna and V. Panferov, Double milling in selfpropelled swarms from kinetic theory, Kinetic and Related Models 2 (2009) 363-378.

[8] J. A. Carrillo, M. Fornasier, J. Rosado and G. Toscani, Asymptotic Flocking Dynamics for the kinetic Cucker-Smale model, preprint UAB, .

[9] J. A. Carrillo and G. Toscani, Contractive probability metrics and asymptotic behavior of dissipative kinetic equations, Riv. Mat. Univ. Parma 6 (2007) 75-198.

[10] Y.-L. Chuang, M. R. D’Orsogna, D. Marthaler, A. L. Bertozzi and L. Chayes, State transitions and the continuum limit for a 2D interacting, self-propelled particle system, Physica D 232 (2007) 33-47.

[11] I.D. Couzin, J. Krause, N.R. Franks and S.A. Levin, Effective leadership and decision making in animal groups on the move, Nature 433 (2005) $513-516$.

[12] F. Cucker and S. Smale, On the mathematics of emergence, Japan. J. Math. 2 (2007) 197-227.

[13] F. Cucker and S. Smale, Emergent behavior in flocks, IEEE Trans. Automat. Control 52 (2007) 852-862.

[14] R. Dobrushin, Vlasov equations, Funct. Anal. Appl. 13 (1979) 115-123.

[15] M.R.D'Orsogna, Y.-L. Chuang, A. L. Bertozzi, L. Chayes, Self-propelled particles with soft-core interactions: patterns, stability, and collapse, Phys. Rev. Lett. 96 (2006) 104302-1/4.

[16] R. Eftimie, G. de Vries, M.A. Lewis, Complex spatial group patterns result from different animal communication mechanisms, Proceedings of the National Academy of Sciences 104 (2007) 6974-6979.

[17] F. Golse, The Mean-Field Limit for the Dynamics of Large Particle Systems, Journées équations aux dérivées partielles 9 (2003) 1-47.

[18] G. Grégoire and H. Chaté, Onset of collective and cohesive motion, Phy. Rev. Lett. 92 (2004) 025702-1/4. 
[19] S.-Y. Ha and J.-G. Liu, A simple proof of the Cucker-Smale flocking dynamics and mean-field limit, to appear in Comm. Math. Sci.

[20] S.-Y. Ha and E. Tadmor, From particle to kinetic and hydrodynamic descriptions of flocking, Kinetic and Related Models 1 (2008) 415-435.

[21] M. Hauray and P.-E. Jabin, $N$-particles approximation of the Vlasov equations with singular potential, Arch. Ration. Mech. Anal. 183 (2007) 489524.

[22] A.L. Koch, D. White, The social lifestyle of myxobacteria, Bioessays 20 (1998) 1030-1038.

[23] H. Levine, W.-J. Rappel and I. Cohen, Self-organization in systems of self-propelled particles, Phys. Rev. E 63 (2000) 017101-1/4.

[24] Y.X. Li, R. Lukeman and L. Edelstein-Keshet, Minimal mechanisms for school formation in self-propelled particles, Physica D 237 (2008) 699720 .

[25] R. Lukeman, Y.X. Li and L. Edelstein-Keshet, A conceptual model for milling formations in biological aggregates, Bull Math Biol. 71 (2008) $352-382$.

[26] A. Mogilner, L. Edelstein-Keshet, L. Bent and A. Spiros, Mutual interactions, potentials, and individual distance in a social aggregation, J. Math. Biol. 47 (2003) 353-389.

[27] H. Neunzert, The Vlasov equation as a limit of Hamiltonian classical mechanical systems of interacting particles, Trans. Fluid Dynamics 18 (1977) 663-678.

[28] H. Neunzert, An introduction to the nonlinear Boltzmann-Vlasov equation, in Kinetic theories and the Boltzmann equation (Montecatini, 1981), pp. 60-110, Lecture Notes in Math. 1048, (Springer, Berlin, 1984).

[29] J. Parrish, L. Edelstein-Keshet, Complexity, pattern, and evolutionary trade-offs in animal aggregation, Science 294 (1999) 99-101.

[30] L. Perea, G. Gómez, and P. Elosegui, Extension of the Cucker-Smale control law to space flight formations, AIAA Journal of Guidance, Control, and Dynamics 32 (2009) 527-537.

[31] H. Spohn, Kinetic equations from Hamiltonian dynamics: Markovian limits, Rev. Modern Phys. 52 (1980) 569-615.

[32] H. Spohn, Large scale dynamics of interacting particles, Texts and Monographs in Physics, Springer 1991. 
[33] C.M. Topaz and A.L. Bertozzi, Swarming patterns in a two-dimensional kinematic model for biological groups, SIAM J. Appl. Math. 65 (2004) $152-174$.

[34] C.M. Topaz, A.L. Bertozzi and M.A. Lewis, A nonlocal continuum model for biological aggregation, Bulletin of Mathematical Biology 68 (2006) $1601-1623$.

[35] T. Vicsek, A. Czirok, E. Ben-Jacob, I. Cohen and O. Shochet, Novel type of phase transition in a system of self-driven particles, Phys. Rev. Lett. 75 (1995) 1226-1229.

[36] C. Villani, Topics in optimal transportation, Graduate Studies in Mathematics Vol. 58, (Amer. Math. Soc, Providence, 2003). 